\title{
Biochemical remission by chemoradiotherapy in male mediastinal choriocarcinoma with diffuse lung metastasis: A case report
}

\author{
JING ZHANG, ZHI-JUN WANG, BIN YANG, YOU-YING WEI, LING YANG, YANG HU and YAN-PING HU \\ Department of Medical Oncology, Hubei Cancer Hospital, Wuhan, Hubei 430079, P.R. China
}

Received January 3, 2015; Accepted January 29, 2016

DOI: $10.3892 / 01.2016 .4248$

\begin{abstract}
Primary mediastinal choriocarcinoma is a rare malignancy that is characterized by multiple metastases at the time of diagnosis, poor response to therapy and short survival times. There is no standard treatment for this disease. The present study described the case of a 25-year-old man with metastatic mediastinal choriocarcinoma. The patient completed 8 cycles of standard combination chemotherapy consisting of etoposide $\left[100 \mathrm{mg} / \mathrm{m}^{2}\right.$; intravenous (IV) drip on days 1-3], cisplatin (20 mg/m² ;V drip on days 1-5) and bleomycin $\left(20 \mathrm{mg} / \mathrm{m}^{2}\right.$; intramuscular injection on days 1 , 8 and 15 every 21 days). The $\alpha$-fetoprotein level decreased to $2.36 \mathrm{ng} / \mathrm{ml}$, the serum $\beta$-human chorionic gonadotropin ( $\beta$-HCG) level markedly decreased to $8.69 \mathrm{IU} / 1$, which was slightly higher than the normal upper limit, and the lactate dehydrogenase level decreased to a normal range. The computed tomography $(\mathrm{CT})$ scan revealed that the number and size of the lung lesions was significantly reduced subsequent to 8 cycles of chemotherapy and the size of the mediastinal tumor was evidently reduced, with a less solid component and a more cystic component. The response assessment indicated partial remission. Following chemotherapy, a radiation dose of $50 \mathrm{~Gy}(2.0 \mathrm{~Gy} /$ fraction) was administered to the involved field of the mediastinum. Following radiotherapy, the $\beta$-HCG level had also decreased to normal levels, and CT evaluation revealed that the size of the residual lung lesions demonstrated no evident change, and the mediastinal tumor was slightly reduced in size, with a less solid component. The patient refused to undergo surgery and did not receive additional treatment following radiotherapy. At present, the patient has survived $>16$ months of follow-up without any symptoms.
\end{abstract}

Correspondence to: Dr Yan-Ping $\mathrm{Hu}$, Department of Medical Oncology, Hubei Cancer Hospital, 116 Zhuo Dao Quan South Road, Wuhan, Hubei 430079, P.R. China

E-mail: hu_yanping1962@126.com

Key words: biochemical remission, chemotherapy, lung metastasis, primary mediastinal choriocarcinoma, radiotherapy

\section{Introduction}

Extragonadal choriocarcinoma is a rare malignancy that occurs mainly in men, and accounts for $1-5 \%$ of all germ cell malignancies $(1,2)$. Chemotherapy, radiotherapy and surgery are the primary therapeutic strategies for treating this disease; however, a large number of patients rapidly succumb to the illness (3-5). A recent study stated that following surgery and chemotherapy the median survival time of 13 male patients with extragonadal choriocarcinoma was 54 months, which was higher than previously expected (6). The most common primary site is the mediastinum (7). The majority of cases are characterized by multiple metastases at the time of diagnosis, poor response to therapy and short survival times $(2,8,9)$. The pathogenesis of extragonadal choriocarcinoma has long been debated and is now focused on two hypotheses (2). One is that the tumor arises from retained primordial germ cells that migrate abnormally during embryogenesis (10). The other is that the lesion originates from the transformation of a nontrophoblastic neoplasm (11).

Serum $\beta$-human chorionic gonadotropin $(\beta-\mathrm{HCG})$ is a classic trophoblastic marker (12). The levels of $\beta-\mathrm{HCG}$, as assessed prior to chemotherapy, have been integrated into the germ cell tumor classification (13). Normalization of high $\beta$-HCG levels is generally associated with a good response to therapy (14). The present study describes the case of a man with metastatic mediastinal choriocarcinoma, in which biochemical remission was achieved by the combination of chemotherapy and radiotherapy.

\section{Case report}

A 25-year-old man was admitted to the Department of Medical Oncology, Hubei Cancer Hospital (Wuhan, Hubei, China) in August 2013 with a month-long history of vomiting, heart palpitations, chest pain and progressing dyspnea. The computed tomography (CT) scan (SOMATOM Definition AS; Siemens AG, Munich, Germany) of the thorax showed a large anterior mediastinal mass and bilateral multiple rounded intrapulmonary lesions, which are characteristic of metastatic tumors. The physical examination was normal, with the exception of bilateral hypermastia. The testicles were equal in size and without nodules or masses. In order to elucidate the feature and distribution of the lesions, a fluoro-2-deoxy-D-glucose (FDG) positron emission 
A

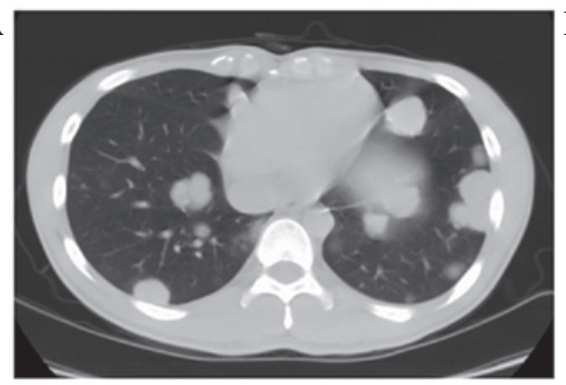

C

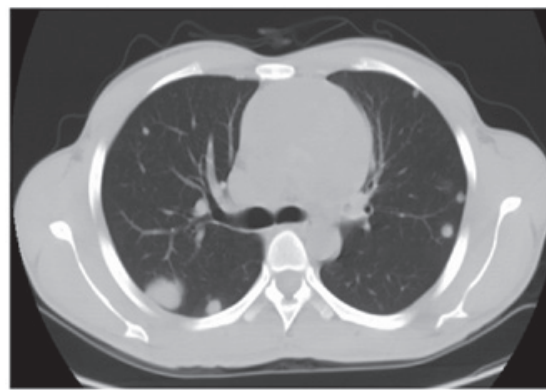

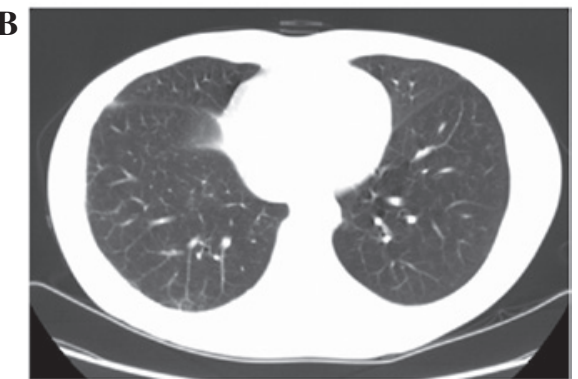

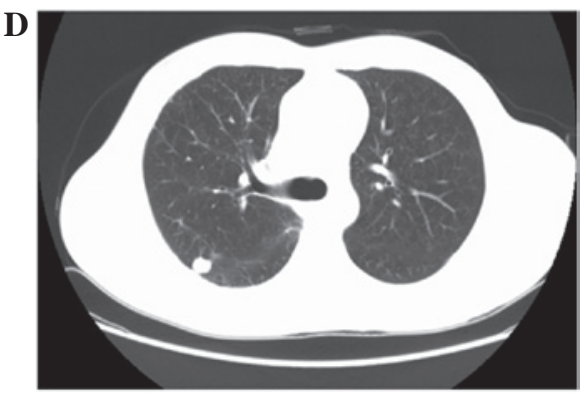

Figure 1. Transverse section of thoracic CT of the lung window prior to and subsequent to chemotherapy. (A) Bilateral multiple lung lesions prior to chemotherapy. (B) The lung lesions present in Fig. 1A are not present in the CT performed subsequent to 8 cycles of chemotherapy. (C) Several lung lesions in another CT section prior to chemotherapy. (D) A small nodule remains in the same CT section of the right lung observed in Fig. 1C subsequent to 8 cycles of chemotherapy. CT, computed tomography.
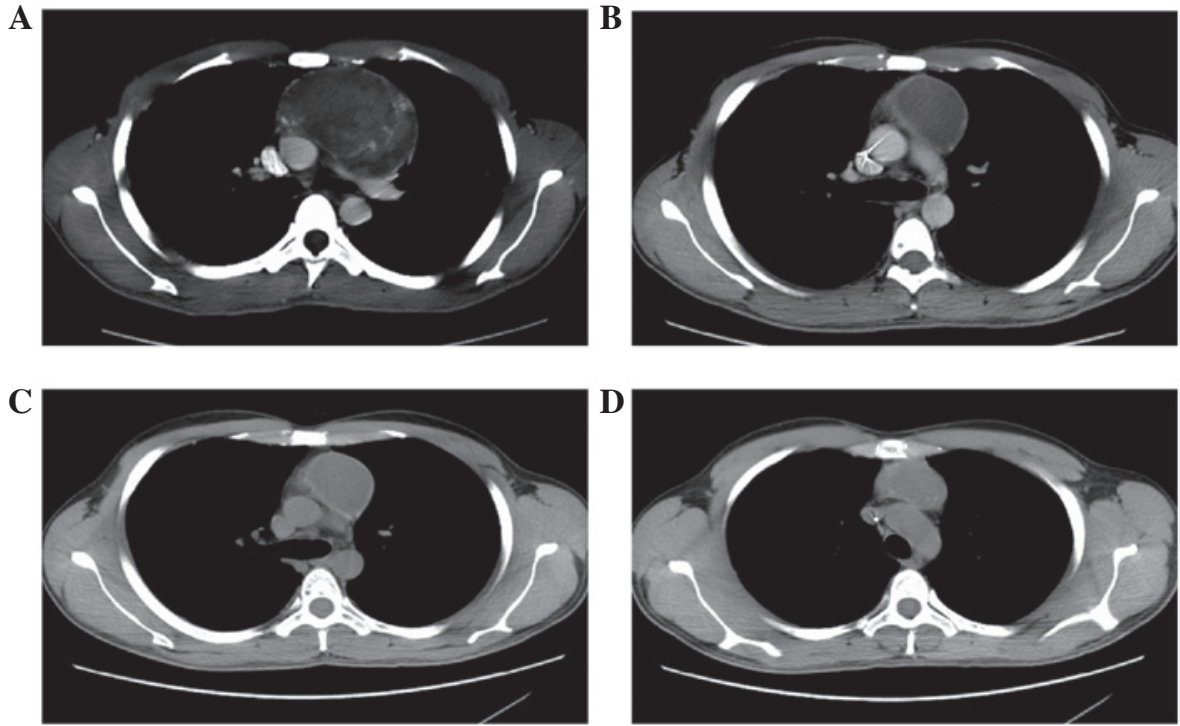

Figure 2. Transverse section of thoracic CT of the mediastinal window during treatment. (A) A large cystic-solid mixed mass was present in the anterior mediastinum prior to chemotherapy. (B) The mass identified in Fig. 1A is evidently reduced in size subsequent to 8 cycles of chemotherapy. (C) The mass in the same CT section as Fig. 1B is slightly reduced in size subsequent to radiotherapy. (D) A markedly less solid component remains in another CT section of the mediastinal mass subsequent to radiotherapy. CT, computed tomography.

tomography (PET)/CT scan (Discovery 16STE; GE Healthcare Life Sciences, Chalfont, UK) was performed. The scan revealed increased FDG uptake in the anterior mediastinal and lung lesions, with a maximal standard uptake value of 8.4 and no extra lesions.

Laboratory data revealed a normal complete blood cell count, and normal hepatic and renal function. However, the levels of the serum tumor markers were elevated, with the exception of carcinoembryonic antigen $(1.20 \mu \mathrm{g} / \mathrm{l})$, as follows: Serum $\beta$-HCG, 10883.2 IU/l (normal, <5 IU/l); $\alpha$-fetoprotein (AFP), $23.1 \mathrm{ng} / \mathrm{ml}$ (normal, $<7 \mathrm{ng} / \mathrm{ml}$ ); and lactate dehydrogenase (LDH), $462 \mathrm{U} / 1$ (normal, <240 U/l). To avoid the risks associated with mediastinal biopsy, including hemorrhage and rupture, an ultrasonography-guided biopsy of the left lung mass was performed, which revealed histological features of a cluster of atypical cells near a small number of squamous epithelium cells, and muscle and alveolar tissue, with the presence of cytotrophoblasts and syncytiotrophoblasts.

Immunohistochemically, the tumor cells were positive for HCG, pan-cytokeratin (CK) and CK7, and did not express cluster of differentiation 117, prostatic acid phosphatase, CK5/6, 
vimentin, leukocyte common antigen or thyroid transcription factor-1. Based on these findings, a diagnosis of primary mediastinal choriocarcinoma with diffuse lung metastasis was made. The patient completed 8 cycles of standard combination chemotherapy that consisted of etoposide $\left[100 \mathrm{mg} / \mathrm{m}^{2}\right.$, intravenous (IV) drip d1-3], cisplatin (20 mg/m², IV drip d1-5) and bleomycin $\left(20 \mathrm{mg} / \mathrm{m}^{2}\right.$ intramuscular injection $\mathrm{d} 1, \mathrm{~d} 8$, d15, Q21d) (BEP) between September 2013 and April 2014, without experiencing notable toxicity. Neutropenia grade I-III, according to the National Cancer Institute-Common Terminology Criteria for Adverse Events v3.0 (15), was the main adverse effect experienced by the patient. During the treatment period, the AFP level decreased to $2.36 \mathrm{ng} / \mathrm{ml}$, which was in the normal range, and $\beta$-HCG markedly decreased to $8.69 \mathrm{IU} / 1$, which was slightly higher than the normal upper limit. LDH decreased to $144 \mathrm{U} / 1$, which was in the normal range. Dynamic CT evaluation revealed that the number and size of the lung lesions was significantly reduced subsequent to 8 cycles of chemotherapy (Fig. 1A-D), and the size of the mediastinal tumor was evidently reduced, with a less solid component and a more cystic component (Fig. 2A-B). The response assessment consistently indicated partial remission, according to Response Evaluation Criteria in Solid Tumors v1.1 (16), every 2 cycles. When chemotherapy was completed, the patient continued to experience left chest pain without dyspnea. To obtain a better response, a radiation dose of 50 Gy was administered to the involved field of the mediastinum by image-guided radiation therapy. The patient was treated with 2.0 Gy a day and 5 fractions each week. Following radiotherapy, the $\beta$-HCG level had also decreased to a normal level of $3.49 \mathrm{IU} / 1$. CT evaluation showed that the size of the residual lung lesions had not evidently changed, and the mediastinal tumor was slightly reduced in size (Fig. 2C), with a much less solid component (Fig. 2D). The patient refused to undergo surgery and did not receive additional treatment following radiotherapy. At present, the patient has survived $>16$ months of follow-up without any symptoms, with the last evaluation performed on December 18, 2014.

\section{Discussion}

The prognosis of mediastinal choriocarcinoma appears poor. Numerous patients succumb in a period of several weeks to a few months $(2,3,8,9,17)$. An investigation based on 97 patients with male choriocarcinoma revealed that the median overall survival (OS) time was 7.7 months and the 6-month mortality rate was $45.4 \%$ (7). There was a consistent phenomenon among patients with poor prognoses that $\beta-\mathrm{HCG}$ rapidly decreased to a lower level during the treatment and then sharply rose when disease relapsed (17). However, certain studies have reported good results in male choriocarcinoma. A clinical analysis of 13 men with primary choriocarcinoma indicated that the median OS time was 54 months (range, 6-115 months), and the 1- and 3-year survival rates were 53.8 and $43.1 \%$, respectively, although the prognosis was worse compared with the prognosis of gestational choriocarcinoma (6). Kathuria et al (18) reported the case of a patient with mediastinal choriocarcinoma that remained alive and asymptomatic 2 years subsequent to surgery and chemotherapy. A regular occurrence in long-term survivors that suffered from mediastinal choriocarcinoma was identified as markedly elevated $\beta$-HCG levels that decreased to the normal range, without signs of rising again, accompanied by complete clinical remission (6). In the present study, the patient experienced a biochemical remission and had a relatively long survival time, though a small solid component remained in the residual cystic mediastinal mass and two small lesions remained in the lung. To the best of our knowledge, the present study is the first to indicate that biochemical remission may not be associated with clinical remission in male mediastinal choriocarcinoma. The reason for this phenomenon is remains to be elucidated. A possible reason is that the mediastinal mass may be involved in the concurrent development of other germ cell tumors that are resistant to chemotherapy and radiotherapy. However, this may not explain the normalization of all tumor markers.

Primary mediastinal choriocarcinoma is an aggressive and fatal cancer, and therefore rapid and appropriate treatment is essential (2). However, there is no standard management for this disease. Bokemeyer et al (19) suggested that the management principle for mediastinal choriocarcinoma was the same as that of metastatic testicular germ cell tumors. Surgery is generally not the primary treatment for mediastinal choriocarcinoma, due to distant metastasis at initial diagnosis or surgical difficulties, and is only performed if an emergent event associated with the tumor exists, such as hemothorax (20). Chemotherapy is generally administered as the primary initial treatment for male choriocarcinoma (9). Numerous studies had tried various chemotherapy regimens, such as methotrexate/actinomycin/chlorambucil and 5-fluorouracil/leucovorin/oxaliplatin, but these were shown to be unsuccessful in this disease $(21,22)$. The most frequent systemic treatment is the BEP regimen. This combination chemotherapy produces a poor response in numerous patients; however, it shows potential for improving survival in certain patients $(3,23-26)$. Few studies have reported the use of radiotherapy in mediastinal choriocarcinoma. Gaude et al (3) suggested that deep radiation therapy was not effective, as choriocarcinoma is a radioresistant tumor. The patient in the present study refused a second biopsy and surgery, so radiotherapy was then used to control the residual tumor in order to improve the therapeutic effect. This demonstrated that radiation has a mild effect on mediastinal choriocarcinoma. The present results support the good response of the combination of chemotherapy and radiotherapy in this malignancy.

In conclusion, there is no standard treatment for mediastinal choriocarcinoma. BEP remains the optimal chemotherapy regimen for this fatal disease, and multidisciplinary treatment may improve its prognosis. However, it is necessary to explore new treatment for patients with refractory mediastinal choriocarcinoma.

\section{Acknowledgements}

The authors thank all nurses, pathologists and laboratory technicians involved in the present study.

\section{References}

1. Collins DH and Pugh RC: Classification and frequency of testicular tumours. Br J Urol 36: 1-11, 1964. 
2. Moran CA and Suster S: Primary mediastinal choriocarcinomas: A clinicopathologic and immunohistochemical study of eight cases. Am J Surg Pathol 21: 1007-1012, 1997.

3. Gaude GS, Patil P, Malur PR, Kangale R, Dhorigol V, Anurshetru S and Karanji J: Primary mediastinal choriocarcinoma. South Asian J Cancer 2: 79, 2013.

4. Lynch MJG and Blewitt GL: Choriocarcinoma arising in the male mediastinum. Thorax 8: 157-161, 1953.

5. Ramia JM, Alcalde J, Dhimes P and Cubedo R: Metastasis from choriocarcinoma of the mediastinum producing acute appendicitis. Dig Dis Sci 3: 332-334, 1998.

6. Jiang F, Xiang Y, Feng FZ, Ren T, Cui ZM and Wan XR: Clinical analysis of 13 males with primary choriocarcinoma and review of the literature. OncoTargets Ther 7: 1135-1141, 2014

7. Yokoi K, Tanaka N, Furukawa K, Ishikawa N, Seya T, Horiba K, Kanazawa Y, Yamada T, Ohaki Y and Tajiri T: Male choriocarcinoma with metastasis to the jejunum: A case report and review of the literature. J Nippon Med Sch 75: 116-121, 2008

8. Krema H, Navajas E, Simpson ER and Payne D: Choroidal metastasis from a mediastinal choriocarcinoma in a male. Can J Ophthalmol 46: 551-552, 2011.

9. Shen HH, Zhang GS and Xu F: Primary choriocarcinoma in the anterior mediastinum in a man: A case report and review of the literatures. Chin Med J (Engl) 117: 1743-1745, 2004.

10. Fine G, Smith RW Jr and Pachter MR: Primary extragenital choriocarcinoma in the male subject. Case report and review of the literature. Am J Med 32: 776-794, 1962.

11. Deshpande JR and Kinare SG: Choriocarcinomatous transformation in metastases of an anaplastic lung carcinoma-a case report. Indian J Cancer 24: 161-166, 1987.

12. Szturmowicz M, Slodkowska J, Zych J, Rudzinski P, Sakowicz A and Rowinska-Zakrzewska E: Frequency and clinical significance of beta-subunit human chorionic gonadotropin expression in non-small cell lung cancer patients. Tumour Biol 20: 99-104, 1999.

13. Tscherry G, Jacky E, Jost LM and Stahel RA: Risk-adapted chemotherapy of germ cell tumors with carboplatin, etoposide and bleomycin for low-risk and cisplatin, etoposide and ifosfamide for high-risk patients. A single-center study. Oncology 59: 110-117, 2000.

14. Oshima J, Uemura M, Kato T, Nagahara A, Kiuchi H, Tsujimura A and Nonomura N: Modified BEP regimen leads to abatement of choriocarcinoma syndrome in a patient with extra gonadal germ cell tumor: A case report. Hinyokika Kiyo 60: 183-187, 2014 (In Japanese).
15. US Department of Health and Human Services. Common Terminology Criteria for Adverse Events (CTCAE). Version 3. National Institute of Health, National Cancer Institute, Washington, DC, 2006

16. Eisenhauer EA, Therasse P, Bogaerts J, Schwartz LH, Sargent D, Ford R, Dancey J, Arbuck S, Gwyther S, Mooney M, et al: New response evaluation criteria in solid tumours: Revised RECIST guideline (version 1.1). Eur J Cancer 45: 228-247, 2009.

17. Belliveau RE, Wiernik PH and Sickles EA: Blood carcinoembryonic antigen, Regan isoenzyme, and human chorionic gonadotrophin in a man with primary mediastinal choriocarcinoma. Lancet 1: 22-24, 1973.

18. Kathuria S and Jablokow VR: Primary choriocarcinoma of mediastinum with immunohistochemical study and review of the literature. J Surg Oncol 34: 39-42, 1987.

19. Bokemeyer C, Nichols CR, Droz JP, Schmoll HJ, Horwich A, Gerl A, Fossa SD, Beyer J, Pont J, Kanz L, et al: Extragonadal germ cell tumors of the mediastinum and retroperitoneum: Results from an international analysis. J Clin Oncol 20: 1864-1873, 2002.

20. Ruan Z, Wang S, Wang Z and Jing Y: A rare case of bilateral massive hemothorax from spontaneous rupture of a primary mediastinal mixed germ cell tumor. Ann Thorac Surg 93: 664-666, 2012

21. Yoon JH, Kim MS, Kook EH, Ahn SH, Jeong SY, Han MS, Huh JK, Kang HJ, Na II, Cho SY, et al: Primary gastric choriocarcinoma: Two case reports and review of the literatures. Cancer Res Treat 40: 145-150, 2008.

22. Cohen BA and Needle MA: Primary mediastinal choriocarcinoma in a man. Chest 67: 106-108, 1975.

23. Shastri A, Daver NG and Hayes TG: Primary gastric chorioadenocarcinoma: A needle in a haystack. Rare Tumors 3: e19, 2011.

24. Zhang F, Zhang W, Shi H, Ye G, Shi W, Shu Y and Li G: Primary choriocarcinoma of the posterior mediastinum in a male: A case report and review of the literature. Oncol Lett 8: 739-741, 2014.

25. Liu Z, Mira JL and Cruz-Caudillo JC: Primary gastric choriocarcinoma: A case report and review of the literature. Arch Pathol Lab Med 125: 1601-1604, 2001.

26. Yamane T, Egawa H, Deguchi N and Moritani C: A case of primary mediastinal choriocarcinoma. Nihon Kokyuki Gakkai Zasshi 44: 48-54, 2006 (In Japanese). 